\title{
ON THE COMMUTATIVE PROPERTIES OF BOUNDARY INTEGRAL OPERATORS
}

\author{
G. F. ROACH
}

\begin{abstract}
A discussion of the interior Dirichlet and Neumann problems of classical potential theory can be given in terms of the symmeterisers of certain related integral operators. Recent developments in the theory and application of integral equations of the first kind have made this approach towards the solution of boundary value problems a more attractive proposition. However for problems more general than those arising in potential theory a greater knowledge of associated spectral properties is required together with a realisation that much of the symmetry occurring in potential problems will be lost and that attention must be directed instead towards commutativity relations. This is demonstrated by considering boundary value problems associated with the Helmholtz equation.
\end{abstract}

1. Introduction. Let $H$ be a complex separable Hilbert space with inner product denoted by $(\cdot, \cdot)$. A linear operator $L: H \rightarrow H$ with domain $D(L)$ dense in $H$ has an associated adjoint $L^{*}: H \rightarrow H$ with domain $D\left(L^{*}\right)$. The operator $L$ is selfadjoint when $L=L^{*}$ and symmetric when $L \subset L^{*}$.

A symmetric operator $L: H \rightarrow H(M: H \rightarrow H)$ will be called a left (right) symmeteriser of a linear operator $K: H \rightarrow H$ whenever the operator product $L K$ (respectively $K M$ ) is a symmetric operator. If $L$ is a left symmeteriser of $K$ then $L K=K^{*} L$ and it is clear that whenever $L^{-1}$ exists then it will satisfy the relation $K L^{-1}=L^{-1} K^{*}$. This indicates that the inverse of a left (right) inverse of an operator $K$ should be sought amongst the right (left) symmeterisers of $K$.

These concepts have been applied by Howland [2] to a discussion of the interior Dirichlet and Neumann problems of classical potential theory. However, a similar application to more general boundary value problems does not seem to have been made. This is due, at least in part, to the fact that in [2] boundary integral equations of the first kind rather than the second kind had to be solved. Furthermore, it is readily appreciated that in more general problems the eigenvalue spectrum of the operators involved is likely to exert a far greater influence on the required analysis than it did in the problems considered in [2]. Recent developments in the theory and application of integral equations of the first kind [3], [5], [4] have made the

Received by the editors March 21, 1978.

AMS (MOS) subject classifications (1970). Primary 35J25.

Key words and phrases. Boundary integral operators.

(c) 1979 American Mathematical Society 0002-9939/79/0000-0066/\$03.25 
approach towards the solution of boundary value problems by means of such equations a more attractive proposition. Consequently it should be possible to obtain somewhat similar results to those obtained in [2] provided there exists enough detailed information on the interaction of the various associated spectra. However we shall see that in these more general problems, much of the symmetry appearing in [2] is lost and our attention has, instead, to be directed towards establishing commutativity relations between the various operators involved. In this note, we shall demonstrate this aspect by considering the four regular boundary value problems (interior and exterior Dirichlet and Neumann problems) associated with the Helmholtz equation since the interaction of their spectra has been comprehensively discussed in [6].

2. Statement of the problems. Let $D_{-}$denote a bounded, connected domain in $\mathbf{R}^{n}$ with boundary $\partial D$ a closed bounded Lyapunov surface. We denote the exterior of $\partial D$, namely the complement of $\bar{D}_{-}:=D_{-} \cup \partial D$ in $\mathbf{R}^{n}$, by $D_{+}$. Let $L$ denote a typical second order, linear, elliptic formally selfadjoint partial differential expression defined in $\mathbf{R}^{n}$. A fundamental solution of the equation $L u=0$ is a two point function of position denoted by $\gamma(P, Q)$ where $P$ and $Q$ are typical points in $\mathbf{R}^{n}$ with coordinates appropriately subscripted.

Typical of the regular boundary value problems associated with $L$ is the Interior Neumann Problem (INP) which consists of determining $u(P)$ such that

$$
\begin{gathered}
(L u)(P)=0, \quad P \in D_{-}, \\
\partial u(p) / \partial n_{p}=g(p), \quad p \in \partial D,
\end{gathered}
$$

where $\partial / \partial n_{p}$ denotes differentiation in the direction of $\hat{n}_{p}$ the outward drawn unit normal to $\partial D$ at $p \in \partial D$. Furthermore we shall write $\partial / \partial n_{p}^{-}$and $\partial / \partial n_{p}^{+}$ to denote the normal derivative when $P \rightarrow p^{-} \in \partial D$ from $D_{-}$and $P \rightarrow p^{+}$ $\in \partial D$ from $D_{+}$respectively.

The Interior Dirichlet Problem (IDP) consists in determining $u(P)$ which solves (2.1) and satisfies the condition

$$
u(p)=f(p), \quad p \in \partial D .
$$

Similar problems, the Exterior Neumann Problem (ENP) and Exterior Dirichlet Problem (EDP), can be posed in $D_{+}$provided that some suitable radiation conditions are adjoined to the problem [1].

3. On the representation of solutions. In order to fix ideas consider problems associated with the three dimensional Helmholtz equation. In this case we set $n=3$,

$$
L=\Delta+k^{2}, \quad k^{2}>0
$$

and

$$
\gamma(P, Q)=-e^{i k R} / 2 \pi R, \quad R=|P-Q|, \quad P, Q \in \mathbf{R}^{3}
$$


Using the Helmholtz representation for the solution of problems posed in $D_{+}$and $D_{-}$we obtain [6]

$$
\begin{aligned}
& \left(S \frac{\partial \phi_{+}}{\partial n}\right)(P)-\left(D \phi_{+}\right)(P)= \begin{cases}2 \phi_{+}(P), & P \in D_{+}, \\
\phi_{+}(p), & p \in \partial D, \\
0, & P \in D_{-},\end{cases} \\
& \left(D \phi_{-}\right)(P)-\left(S \frac{\partial \phi_{-}}{\partial n}\right)(P)= \begin{cases}0, & P \in D_{+}, \\
\phi_{-}(p), & p \in \partial D, \\
2 \phi_{-}(P), & P \in D_{-},\end{cases}
\end{aligned}
$$

where $\phi_{+}\left(\phi_{-}\right)$is the solution of the problem posed in $D_{+}\left(D_{-}\right)$and

$$
\begin{aligned}
& (S \mu)(P)=\int_{\partial D} \mu(q) \gamma(P, q) d S_{q}, \quad P \in D_{ \pm}, \\
& (D v)(P)=\int_{\partial D} \nu(q) \frac{\partial \gamma}{\partial n_{q}}(P, q) d S_{q}, \quad P \in D_{ \pm} .
\end{aligned}
$$

We introduce the notation

$$
\begin{aligned}
(K \mu)(p) & :=\int_{\partial D} \mu(q) \frac{\partial \gamma}{\partial n_{p}}(p, q) d S_{q}, \quad p, q \in \partial D, \\
\left(K^{*} \mu\right)(p) & :=\int_{\partial D} \mu(q) \frac{\partial \bar{\gamma}}{\partial n_{q}}(p, q) d S_{q}, \quad p, q \in \partial D, \\
\left(D_{n} \nu\right)(p) & :=\frac{\partial}{\partial n_{p}} \int_{\partial D} \nu(q) \frac{\partial \gamma}{\partial n_{q}}(p, q) d S_{q} \\
& =\frac{\partial}{\partial n_{p}}\left(\left(K^{*} v\right)(p)\right), \quad p, q \in \partial D,
\end{aligned}
$$

where the bar denotes complex conjugate and * the formal $L_{2}(\partial D)$ adjoint. In the limit as $P \rightarrow p \in \partial D$ the various jump conditions [6] can be written

$$
\begin{aligned}
\partial(S \mu)(p) / \partial n_{p}^{ \pm} & = \pm \mu(p)+(K \mu)(p), \\
\lim _{P \rightarrow p^{ \pm}}(D v)(p) & =\mp \nu(p)+\left(\bar{K}^{*} \nu\right)(p) .
\end{aligned}
$$

Furthermore, we notice that

$$
(D v)(p)=\left(\bar{K}^{*} \nu\right)(p), \quad p \in \partial D,
$$

and

$$
\frac{\partial}{\partial n_{p}^{+}}(D v)(p)=\frac{\partial}{\partial n_{p}^{-}}(D v)(p)=\left(D_{n} v\right)(p), \quad p \in \partial D .
$$

We now pose two of the regular problems for the Helmholtz equation. 
Interior Neumann Problem (INP).

$$
\begin{aligned}
\left(L u_{-}\right)(P) & :=\left(\Delta+k^{2}\right) u_{-}(P)=0, \quad P \in D_{-}, \\
\partial u_{-}(p) / \partial n_{p} & =g_{-}(p), \quad p \in \partial D .
\end{aligned}
$$

Interior Dirichlet Problem (IDP).

$$
\begin{aligned}
\left(L v_{-}\right)(P) & :=\left(\Delta+k^{2}\right) v_{-}(P)=0, \quad P \in D_{-}, \\
v_{-}(p) & =f_{-}(p), \quad p \in \partial D .
\end{aligned}
$$

These problems can be reformulated as boundary integral equations either by applying the boundary conditions together with the appropriate jump conditions to the Helmholtz representation or by assuming that the required solution has the form of a single or double layer distribution. The intention in each case is to obtain a boundary integral equation of the second kind, although it can be shown [6] that these two methods yield different equations of the second kind for the same problem.

Similar problems can also be posed in the exterior domain $D_{+}$. The relationships between the two methods outlined above and the interconnections between the exterior and interior problems have been discussed at some length in [6].

It is common practice in a layer theoretic approach to assume that solutions of Neumann problems have the form of single layer distributions whilst solutions of Dirichlet problems have the form of double layer distributions. In each case such an assumption leads to boundary integral equations of the second kind.

The solutions of the Neumann and Dirichlet problems could however be sought in the form of double and single layer distributions respectively. In this case boundary integral equations of the first kind are obtained. This approach has been adopted by some authors and in this connection we would cite [2] as a source reference. Furthermore, in [2] it is argued that some of the inherent difficulties associated with equations of the first kind might be eased significantly provided certain relationships between an operator and its symmeterisers can be established. These necessary relationships have been obtained in [2] for problems arising in classical potential theory. In the next section we shall illustrate the derivation of the corresponding results for boundary value problems associated with the Helmholtz equation.

4. Commutative properties of boundary integral operators. Using (3.4) the solution of the INP, (3.14) can be represented in the form [6]

$$
u_{-}(P)=\frac{1}{2}\left(D u_{-}\right)(P)-\left(S g_{-}\right)(P), \quad P \in D_{-},
$$

where

$$
u_{-}(p)=\left(D u_{-}\right)(p)-\left(S g_{-}\right)(p), \quad p \in \partial D .
$$

Equation (4.2) must be solved for $u_{-}(p)$ in order that the representation (4.1) should be effective. However, as is emphasised in [6], any solution of (4.2) 
must also satisfy the compatibility condition

$$
\left(D_{n} u_{-}\right)(p)=\left(K g_{-}+g_{-}\right)(p) .
$$

In a layer theoretic approach the solution of the INP can be sought in the form

$$
u_{-}(P)=-(S \mu)(P), \quad P \in D_{-},
$$

where the required density function, $\mu$, must satisfy [6]

$$
(\mu-K \mu)(p)=g_{-}(p), \quad p \in \partial D .
$$

Combining the Green's Theorem and layer theoretic methods we obtain from (4.2), (4.4) and (4.5)

$$
-(S \mu)(p)=-(D(S \mu))(p)-(S(\mu-K \mu))(p)
$$

Using (3.12) in this last result yields the commutativity relationship

$$
\left(\bar{K}^{*} S \mu\right)(p)=(S K \mu)(p), \quad p \in \partial D .
$$

Furthermore, by writing the compatibility condition (4.3) in the form

$$
g_{-}(p)=\left(D_{n} u_{-}\right)(p)-\left(K g_{-}\right)(p)
$$

and using the continuity of a single layer together with (4.4) and (4.5) we obtain

$$
(\mu-K \mu)(p)=-\left(D_{n} S \mu\right)(p)-(K(\mu-K \mu))(p)
$$

Hence

$$
\left(D_{n} S \mu\right)(p)=\left(\left(K^{2}-I\right) \mu\right)(p), \quad p \in \partial D .
$$

The INP can also be solved, using the layer theoretic method, by assuming

$$
u_{-}(P)=(D v)(P), \quad P \in D_{-} .
$$

Acknowledging the relation (3.13) we see that the required density function $\nu$ must satisfy the following equation of the first kind

$$
g_{-}(p)=\left(D_{n} \nu\right)(p), \quad p \in \partial D .
$$

On the other hand, applying (3.11) to (4.8) we obtain

$$
u_{-}(p)=\nu(p)+\left(\bar{K}^{*} \nu\right)(p), \quad p \in \partial D,
$$

where both $\nu$ and $u_{-}$are as yet unknown. However, this difficulty can be eased if we notice that (4.3) holds for all solutions of the INP and so must hold for $u_{-}$given by (4.8). Consequently, applying $D_{n}$ to both sides of (4.10), and substituting in (4.3) we obtain (on acknowledging (4.9))

$$
\left(D_{n} \bar{K}^{*} \nu\right)(p)=\left(K D_{n} \nu\right)(p)
$$

Furthermore, (4.8), (4.9), (3.11) and the continuity of a single layer potential across the boundary of the region of interest enables (4.2) to be written in the form

$$
\left[\left(I+\bar{K}^{*}\right) \nu\right](p)=\left[D\left(I+\bar{K}^{*}\right) \nu\right](p)-\left(S D_{n} \nu\right)(p)
$$


From which it follows that

$$
\left(S D_{n} \nu\right)(p)=\left[\left(\bar{K}^{* 2}-I\right) \nu\right](p)
$$

For the IDP the required solution is given by the Helmholtz representation in the form

$$
v_{-}(P)=\frac{1}{2}\left(D f_{-}\right)(P)-(S w)(P), \quad P \in D_{-},
$$

where

$$
\begin{aligned}
{[(K+I) w](p) } & =\left(D_{n} f_{-}\right)(p), \quad p \in \partial D, \\
(S w)(p) & =\left[\left(\bar{K}^{*}-I\right) f_{-}\right](p), \quad p \in \partial D, \\
w(p) & =\partial v_{-}(p) / \partial n_{p}, \quad p \in \partial D .
\end{aligned}
$$

In the layer theoretic approach to this problem it is usual to look for a solution in the form

$$
v_{-}(P)=(D v)(P), \quad P \in D_{-},
$$

where the density function must satisfy

$$
[(K+I) \nu](p)=f_{-}(p), \quad p \in \partial D .
$$

However, the IDP could also be solved in a layer theoretic approach by assuming that the solution has the form

$$
v_{-}(P)=-(S \mu)(P), \quad p \in D_{-},
$$

which would imply that the required density should satisfy the first kind equation

$$
f_{-}(p)=-(S \mu)(p), \quad p \in \partial D .
$$

Consequently, arguing as for the INP, it is an easy matter to deduce that the relations (4.6), (4.7), (4.11) and (4.12) also hold when discussing the IDP. Furthermore, if use is made of the pairings given in Table 2 of [6] it can be seen that similar results also hold for the corresponding exterior problems.

The results of this section can be summarised in the form:

THEOREM 4.1. The boundary integral operators $S, D, K$ and $D_{n}$ associated with the Helmholtz equation and regarded as mappings of $L_{2}(\partial D)$ into itself have the following commutative properties.

$$
\begin{aligned}
S K & =\overline{K^{*}} S, \quad K D_{n}=D_{n} \bar{K}^{*}, \\
S D_{n} & =\bar{K}^{* 2}-I, \quad D_{n} S=K^{2}-I .
\end{aligned}
$$

We notice that although these results have the same symbolic form as those obtained in [2] nevertheless they do offer a generalisation of the latter in that here $S$ and $D_{n}$ are no longer symmetric. Indeed if we write $S_{k}\left(S_{0}\right)$ for the operator $S$ associated with the Helmholtz (Laplace) equation, and similar subscripts for the other boundary integral operators, then direct computation 
shows that

$$
\begin{aligned}
\left(S_{k} u, v\right) & =\left(u, \bar{S}_{k} v\right) \quad \text { and } \quad\left(S_{0} u, v\right)=\left(u, S_{0} v\right), \\
\left(D_{n k} u, v\right) & =\left(u, \bar{D}_{n k} v\right) \quad \text { and } \quad\left(D_{n 0} u, v\right)=\left(u, D_{n 0} v\right)
\end{aligned}
$$

It is clear that Howland's results on symmeterisers are recovered in the case when $k=0$.

5. Concerning the first kind equations. The results of the previous section rely on our being able to solve equations (4.9) and (4.16). The solvability of these first kind equations, when they arise in connection with boundary value problems of potential theory, has been discussed in [2]. To obtain corresponding details for the similar equations which are obtained when considering problems associated with the Helmholtz equation particular attention must be paid to the influence of the frequency parameter $k$. The spectrum of the operator $K$ is clearly of vital importance and, since $K$ may be regarded as an operator valued function of the parameter $k$ it is convenient to treat separately the operators $(-K)$ and $(+K)$ rather than introduce yet another parameter $\lambda$ in order to obtain the standard form $(I-\lambda K)$. With this in mind we adopt the following definitions. Those real values of $k$ for which the equation $(I-K) w=0$ has nontrivial solutions, $w$, will be called characteristic values of $K$. Similarly, those real values of $k$ for which $(I+K) w=0$ has nontrivial solutions, $w$, will be called characteristic values of $(-K)$. We note that if $k$ is a characteristic value of $K$ then it is also a characteristic value of $K^{*}, \bar{K}$ and $\bar{K}^{*}$. Similarly $(-K),(-\bar{K}),\left(-K^{*}\right),\left(-\bar{K}^{*}\right)$ have the same characteristic values, which may differ from the preceding. Those values of $k$ for which there exist nontrivial solutions of the homogeneous form of the INP (IDP) will be called eigenvalues of the INP (IDP). The relationship between the characteristic values of $K$ and the eigenvalues of interior problems is clarified by the following theorem the proof of which is given in [6].

THEOREM 5.1. The parameter $k$ is a characteristic value of the operator $K$ $(-K)$ if and only if $k$ is an eigenvalue of the INP (IDP).

Regarding the solvability of (4.9) and (4.16) we offer two theorems the proofs of which necessarily rely very heavily on the results obtained in [6].

THEOREM 5.2. Assume that $k$ is not an eigenvalue of either the INP or the IDP. The integral equation (4.16) has a unique solution $\mu \in L_{2}(\partial D)$ for any $f_{-} \in L_{2}(\partial D)$ if and only if $\mu$ satisfies

$$
\left[\left(K^{2}-I\right) \mu\right](p)=\left(D_{n} f_{-}\right)(p), \quad p \in \partial D .
$$

Proof. $(\Rightarrow)$ : Follows immediately from (4.7).

$(\Leftarrow)$ : Assume $f$ an element of the domain $D_{n}$ and that $\mu \in L_{2}(\partial D)$ satisfies 
$\left(K^{2}-I\right) \mu=D_{n} f_{-}$. This implies

$$
\begin{aligned}
(I-K) w & =-D_{n} f_{-}, \\
w & =(I+K) \mu .
\end{aligned}
$$

In [6], Theorem 5.4 states that if $k$ is not an eigenvalue of the INP then (5.2) has a unique solution $w$ for all $f_{-} \in L_{2}(\partial D)$ and moreover this solution satisfies

$$
S w=\left(\bar{K}^{*}+I\right) f_{-} .
$$

However, the uniqueness of $w$ does not, in general, imply the uniqueness of $\mu$ as a solution of (5.3). Indeed if there exist $\mu_{1}$ and $\mu_{2}$ such that

$$
w=(I+K) \mu_{1}=(I+K) \mu_{2}
$$

then

$$
(I+K)\left(\mu_{1}-\mu_{2}\right)=0 .
$$

By Theorem 3.2.1 of [6] we find that (5.5) has a nontrivial solution $\left(\mu_{1}-\mu_{2}\right)$ $=\phi$ if and only if

$$
S_{\phi}=0 .
$$

However, Corollary 3.2.1 of Theorem 3.2.1 in [6] states that (5.6) has a nontrivial solution $\phi$ if and only if $k$ is an eigenvalue of IDP. Therefore as $k$ is not an eigenvalue of either INP or IDP we conclude that $\phi=0$ and hence $\mu$ exists as the unique solution of (5.3).

Combining (5.3) and (5.4) and acknowledging the results of Theorem 4.1 we obtain

$$
\left(I+\bar{K}^{*}\right)\left(S \mu+f_{-}\right)=0 .
$$

Setting $\rho:=(S \mu+f)$ and using Theorem 4.6 of [6] we find that if $\rho$ is a nontrivial solution of $\left(I+\bar{K}^{*}\right) \rho=0$ then $\eta:=D_{n} \rho$ satisfies $(I+K) \eta=0$. However Theorem 3.2.1 and Corollary 3.2.1 of [6] indicate that the solution $\eta$ is nontrivial if and only if $k$ is an eigenvalue of the IDP. Hence we deduce that $D_{n} \rho=0$. Finally, by means of Corollary 3.3.2 in [6] we conclude that the only simultaneous solution of $D_{n} \rho=0$ and $\left(I+\bar{K}^{*}\right) \rho=0$ is the trivial solution $\rho=0$. Hence $S \mu=-f_{-}$as required.

THEOREM 5.3. Assume that $k$ is not an eigenvalue of either the INP or the IDP. The integral equation (4.9) has a unique solution $\nu \in L_{2}(\partial D)$ for any $g_{-} \in L_{2}(\partial D)$ if and only if $\nu$ satisfies the equation

$$
\left[\left(\bar{K}^{* 2}-I\right) \nu\right](p)=\left(S g_{-}\right)(p), \quad p \in \partial D .
$$

Alternatively, (4.9) has a solution $\nu=S \mu$ where $\mu \in L_{2}(\partial D)$ satisfies

$$
\left[\left(K^{2}-I\right) \mu\right](p)=g_{-}(p), \quad p \in \partial D .
$$

Proof. $(\Rightarrow)$ : Follows immediately from (4.12).

$(\Leftarrow)$ : Assume that $g_{-}$an element of the domain of $S$ and that $\nu \in L_{2}(\partial D)$ 
satisfies $\left(\bar{K}^{* 2}-I\right) \nu=S g_{-}$. This implies

$$
\begin{aligned}
\left(\bar{K}^{*}+I\right) w & =S g_{-}, \\
w & =\left(\bar{K}^{*}-I\right) \nu .
\end{aligned}
$$

Since $k$ is not an eigenvalue of the IDP, Theorem 5.1 of [6] implies that (5.7) has a unique solution $w$ for all $g_{-} \in L_{2}(\partial D)$ and moreover this solution satisfies

$$
D_{n} w=(K-I) g_{-} .
$$

If there exist $\nu_{1}$ and $\nu_{2}$ such that

$$
w=\left(\bar{K}^{*}-I\right) \nu_{1}=\left(\overline{K^{*}}-I\right) \nu_{2}
$$

then

$$
\left(\overline{K^{*}}-I\right) \phi=0, \quad \phi=\nu_{1}-\nu_{2} .
$$

Theorem 3.3.1 of [6] indicates that (5.10) has a nontrivial solution $\phi$ if and only if

$$
D_{n} \phi=0 .
$$

In [6], Corollary 3.3.1 of Theorem 3.3.1 states that (5.11) has a nontrivial solution $\phi$ if and only if $k$ is an eigenvalue of the INP. Hence, arguing as in the previous solution we find that $\nu$ exists as the unique solution of (5.8).

Combining (5.8) and (5.9) we obtain

$$
(K-I) \rho=0, \quad \rho=D_{n} \nu-g_{-} .
$$

By Theorem 4.5 of [6] we find that if (5.12) has a nontrivial solution $\rho$ then $\eta:=S \rho$ satisfies the equation $\left(I-\bar{K}^{*}\right)=0$. Using Theorem 3.3.1 of [6] and arguing as in the previous theorem we find that $D_{n} \nu=g_{-}$as required.

Finally, if we set $\nu=S \mu$ then by means of (4.7) we find that

$$
g_{-}=D_{n} \nu=D_{n} S \mu=\left(K^{2}-I\right) \mu \text {. }
$$

\section{REFERENCES}

1. R. A. Adams and G. F. Roach, An intrinsic approach to radiation conditions, J. Math. Anal. Appl. (2) 39 (1972), 433-444.

2. J. L. Howland, Symmeterising kernels and the integral equation of the first kind of classical potential theory, Proc. Amer. Math. Soc. 19 (1968), 1-7.

3. G. C. Hsaio and R. C. McCamy, Solution of boundary value problems by integral equations of the first kind, SIAM Rev. 15 (1973), 687-705.

4. G. C. Hsaio and G. F. Roach, On the relationship between boundary value problems, J. Math. Anal. Appl. (to appear).

5. G. C. Hsaio and W. Wendland, On Galerkin's method for a class of integral equations of the first kind, Applicable Anal. 6 (1977), 155-157.

6. R. E. Kleinman and G. F. Roach, Boundary integral equations for the three dimensional Helmholtz equation, SIAM Rev. 16 (1974), 214-236.

Department of Mathematics, University of Strathclyde, Glasgow, Scotland 